\title{
Accelerated muscle and adipose tissue loss may predict survival in pancreatic cancer patients: the relationship with diabetes and anaemia
}

\author{
Katie M. Di Sebastiano ${ }^{1}$, Lin Yang ${ }^{2}$, Kevin Zbuk ${ }^{2}$, Raimond K. Wong ${ }^{2}$, Tom Chow ${ }^{2}$, David Koff ${ }^{3}$, \\ Gerald R. Moran ${ }^{4}$ and Marina Mourtzakis ${ }^{1 *}$ \\ ${ }^{1}$ Department of Kinesiology, University of Waterloo, 200 University Avenue, West Waterloo, ON, Canada, N2L 3G1 \\ ${ }^{2}$ Department of Oncology, McMaster University, Hamilton, ON, Canada \\ ${ }^{3}$ Department of Radiology, McMaster University, Hamilton, ON, Canada \\ ${ }^{4}$ Lawson Health Research Institute, London, ON, Canada
}

(Submitted 28 September 2011 - Final revision received 11 January 2012 - Accepted 19 February 2012 - First published online 4 July 2012)

\begin{abstract}
Weight loss leading to cachexia is associated with poor treatment response and reduced survival in pancreatic cancer patients. We aim to identify indicators that allow for early detection that will advance our understanding of cachexia and will support targeted anti-cachexia therapies. A total of fifty pancreatic cancer patients were analysed for skeletal muscle and visceral adipose tissue (VAT) changes using computed tomography (CT) scans. These changes were related to physical characteristics, secondary disease states and treatment parameters. Overall, patients lost 1.72 (SD 3.29) kg of muscle and 1.04 (SD 1.08) kg of VAT during the disease trajectory (413 (SD 213 ) d). After sorting patients into tertiles by rate of VAT and muscle loss, patients losing VAT at $>-0 \cdot 40 \mathrm{~kg} / 100 \mathrm{~d}$ had poorer survival outcomes compared with patients with $<-0.10 \mathrm{~kg} / 100 \mathrm{~d}$ of VAT loss $(P=0.020)$. Patients presenting with diabetes at diagnosis demonstrated significantly more and accelerated VAT loss compared with non-diabetic patients. In contrast, patients who were anaemic at the first CT scan lost significantly more muscle tissue and at accelerated rates compared with non-anaemic patients. Accelerated rates of VAT loss are associated with reduced survival. Identifying associated features of cachexia, such as diabetes and anaemia, is essential for the early detection of cachexia and may facilitate the attenuation of complications associated with cachexia.
\end{abstract}

Key words: Cachexia: Muscle wasting: Body composition: Weight loss

The majority of advanced cancer patients experience accelerated involuntary loss of muscle with or without adipose tissue loss during the course of their disease trajectory ${ }^{(1)}$. Weight loss, a defining feature of cachexia, is associated with poorer treatment response and decreased survival in several cancer diagnoses including pancreatic cancer ${ }^{(2)}$. Despite the importance of the interaction between body composition and survival, few studies have characterised specific body composition changes (fat and muscle) using precise measures of muscle and fat such as computed tomography (CT) image analysis. Thus, the relationship between these changes and clinical and physical characteristics is unclear.

A recent international consensus highlighted the importance of identifying key biological predictors of cachexia, such as insulin resistance ${ }^{(3)}$. Body composition is related to a variety of these parameters, and thus, these markers may be potential determinants of muscle and adipose tissue losses. Pancreatic cancer patients experience some of the most severe symptoms of cachexia ${ }^{(4)}$; therefore, the relationships between changes in body composition and physical and clinical characteristics may be more pronounced in this population. Insulin resistance, for example, has been associated with increased muscle wasting ${ }^{(5)}$, suggesting that diabetics may exhibit accelerated rates of muscle wasting when concurrently diagnosed with pancreatic cancer. Anaemia has also been used to diagnose cancer cachexia ${ }^{(6)}$, while surgery has been associated with accelerated tissue loss ${ }^{(7)}$. Thus, these parameters are of particular interest. The potential relationships between body composition and clinical parameters that are documented during routine care, such as inflammatory markers, markers of tumour burden and secondary disease states, have not been evaluated.

Weight and crude measures of fat and lean mass, derived from bioelectrical impedance analysis, are methods commonly

Abbreviations: CSA, cross-sectional area; CT, computed tomography; IMAT, intramuscular adipose tissue; L3, third lumbar vertebrae; SAT, subcutaneous adipose tissue; TAT, total adipose tissue; VAT, visceral adipose tissue.

*Corresponding author: Dr M. Mourtzakis, fax +1 519885 0470, email mmourtza@uwaterloo.ca 
used to examine body composition. For example, Bachmann et $a .^{(2)}$ demonstrated that weight loss is associated with poor survival outcomes in pancreatic cancer patients; however, weight loss measurements do not allow examination of the specific and independent influences of muscle and each of the three major adipose tissue depots (i.e. visceral adipose tissue (VAT), subcutaneous adipose tissue (SAT) and intramuscular adipose tissue (IMAT)).

CT image analysis provides a precise and highly reproducible method to quantify changes in individual fat and muscle tissue depots. In one of the few studies using CT image analysis, Tan et $a l .{ }^{(8)}$ suggested that the proportion of muscle and fat that a patient carried at diagnosis may significantly influence survival. They further noted that muscle and adipose tissue each had distinct patterns of tissue loss; thus, these findings suggest value in examining the independent relationship of muscle and adipose tissue with survival.

Each adipose tissue depot possesses unique metabolic properties; however, VAT may have independent influences on survival and other clinical outcomes. Excessive accumulation of VAT, the most metabolically active adipose tissue ${ }^{(9)}$, is associated with the metabolic syndrome, a risk factor for pancreatic cancer ${ }^{(10)}$. VAT accumulation at cancer diagnosis may also decrease survival time specifically in pancreatic cancer patients ${ }^{(11)}$, suggesting that VAT measurements may be particularly important in understanding survival and other clinical parameters.

The objectives of the present study were to precisely quantify body composition at diagnosis and during the pancreatic cancer trajectory. Subsequently, changes in body composition were related to clinical markers acquired during routine follow-up and clinical outcomes, including survival, to identify associated features of cachexia. Identification of these features is essential for the early detection of cachexia and the attenuation of complications associated with cachexia. We hypothesised that patients would lose significant amounts of muscle and adipose tissue over the disease trajectory. Second, patients with the largest losses and the most accelerated rates of muscle and adipose tissue loss would be associated with the poorest survival outcomes and clinical parameters.

\section{Materials and methods}

\section{Patient selection}

The present study was conducted according to the guidelines laid down in the Declaration of Helsinki and all procedures involving human subjects/patients were approved by the
University of Waterloo Research Ethics Board and the Hamilton Health Sciences Research Ethics Board. Patients diagnosed with pancreatic cancer and who had died between July 2003 and February 2010 were identified at the Juravinski Cancer Centre in Hamilton, ON, Canada, which includes a catchment of approximately 1.2 million people. We classified patients based on stage (I, II, III and IV), progression and treatment. Patients were included if they: (1) died from pancreatic cancer, (2) were diagnosed with either a carcinoma or adenocarcinoma, (3) had at least two abdominal CT scans performed during their disease trajectory and (4) CT scans were performed up to 2 years before their time of death. Patients surviving for less than 4 months were excluded from analysis, as these patients were in the terminal stage of their disease and changes in body composition would have been distinct from patients at earlier stages of the disease $^{(3)}$; these patients would also be less likely to positively respond to neoplastic and nutritional therapies. At this stage of disease, the associated risk of nutritional therapy probably outweighs any potential benefit ${ }^{(3)}$. CT scans that were performed 3 months or more before the date of diagnosis were not included in the present analysis, as they are not representative of body composition during disease presentation. Any two consecutive scans were at least 1 month apart and no longer than 1 year apart (Fig. 1).

\section{Physical and clinical characteristics}

Anthropometric measurements at diagnosis including height and weight were recorded from patient records and used to calculate BMI $\left(\mathrm{kg} / \mathrm{m}^{2}\right)$. Stage of disease at diagnosis was determined using the tumour node metastasis (TNM) classification of malignant tumours system. Survival, stage of tumour, histology and presence of diabetes were recorded at diagnosis only. The presence of anaemia $(\mathrm{Hb}<120 \mathrm{~g} / \mathrm{l}$ for females, $\mathrm{Hb}<130 \mathrm{~g} / 1$ for males) ${ }^{(12)}$, CA19-9 values as well as whether patients received nutritional consults (i.e. patients who met with a registered dietitian regardless of whether nutritional supplements were prescribed) or dietary supplementation (i.e. patients who consumed some form of nutritional supplement in addition to their habitual diet independent of a dietetic consult) were recorded throughout the disease trajectory. $\mathrm{Hb}$ values and CA19-9 values within 1 month of the first CT scan were considered representative of diagnostic values. All patients had $\mathrm{Hb}$ values within that time frame, while only twenty-eight patients had CA19-9 values within that time. These blood parameters were recorded and then related to body composition.

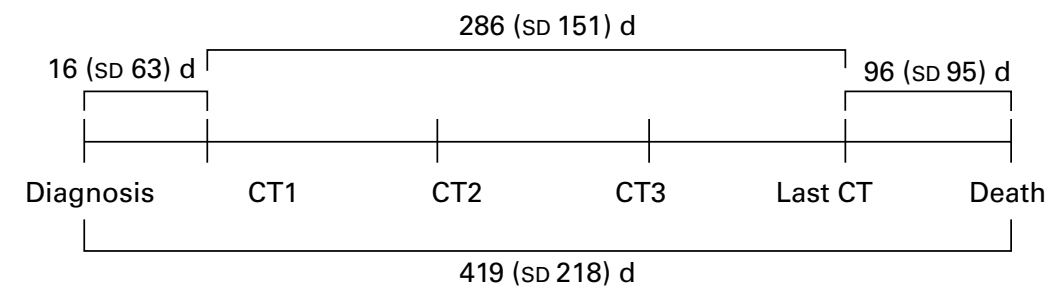

Fig. 1. Representative timeline of the disease trajectory with the average timing of computed tomography (CT) scan. 


\section{Computed tomography image analysis}

Skeletal muscle and adipose tissue cross-sectional areas (CSA) were quantified using single-slice CT scans at the third lumbar vertebrae (L3). Tissue CSA at this location are strongly correlated with whole-body skeletal muscle and adipose tissue mass distribution ${ }^{(13-15)}$. Skeletal muscle tissue included psoas, erector spinae, quadratus lumborum, transverse abdominal, internal and external obliques, and rectus abdominus. We also specifically measured VAT, as it is the most metabolically active fat tissue ${ }^{(9)}$. Importantly, VAT is associated with the metabolic syndrome ${ }^{(16)}$ and pancreatic cancer ${ }^{(10)}$, and it has been associated with decreased survival time in pancreatic cancer patients ${ }^{(11)}$. Thus, VAT may influence clinical outcomes in pancreatic cancer more than other adipose tissue depots.

Slice-O-Matic ${ }^{\circledR}$ version 4.3 (Tomovision) allows the precise quantification of specific tissue using Hounsfield units. Hounsfield unit threshold values were defined at -29 to 150 for skeletal muscle ${ }^{(17)},-150$ to -50 for $\operatorname{VAT}^{(18)}$, and -190 to -30 for both SAT and IMAT ${ }^{(17)}$. CSA $\left(\mathrm{cm}^{2}\right)$ were computed for each tissue by summing tissue pixels and multiplying by the pixel surface area. All CT images were analysed by a single trained analyst who was blinded to clinical markers and patient outcomes. To calculate the intra-analyst CV, five CT images were analysed twice on two separate days 6 months apart. The mean CV for skeletal muscle CSA was 0.322 and $0.130 \%$ for adipose tissue CSA. To avoid bias, two additional trained analysts (who were blinded to both the objectives of the study and the patient characteristics of the CT scans) quantified muscle and adipose tissue for all scans and resulted in a mean $\mathrm{CV}<1.0 \%$ for skeletal muscle CSA and approximately $3.0 \%$ for adipose tissue CSA. Thus, all data presented are from the primary analyst to ensure consistency.

\section{Calculations for estimating tissue mass, net tissue change} and rate of tissue change

There is a strong relationship between $\mathrm{L} 3$ skeletal muscle and whole-body lean tissue in cancer patients $(r 0.83)^{(13)}$; however, equations to predict whole-body skeletal muscle mass from L3 CT images do not exist for this specific population. Therefore, an algorithm derived from a healthy population was used to estimate whole-body skeletal muscle volume from the CSA at $\mathrm{L}^{(13)}$ :

Whole-body skeletal muscle volume $(L)$ $=0.166 \times\left(\right.$ skeletal muscle $5 \mathrm{~cm}$ above $\left.\mathrm{L} 4-\mathrm{L} 5\left(\mathrm{~cm}^{2}\right)\right)$ $+2.142 ; r^{2} 0.855$

where L 4 and L5 are the fourth and fifth lumbar vertebrae, respectively.

A density of $1.04 \mathrm{~g} / \mathrm{cm}^{3}$ was used to estimate skeletal muscle mass $(\mathrm{g})$ from volume ${ }^{(19)}$

Sarcopenia was defined based on cut-off points described in a cohort of advanced cancer patients and defined as $\leq 38.9 \mathrm{~cm}^{2} / \mathrm{m}^{2}$ for females and $\leq 55.4 \mathrm{~cm}^{2} / \mathrm{m}^{2}$ for males ${ }^{(15)}$. To identify whether a patient was below (indicating sarcopenia) or above (indicating non-sarcopenia) these cut-off points, skeletal muscle CSA at L3 $\left(\mathrm{cm}^{2}\right)$ was divided by height squared $\left(\mathrm{m}^{2}\right)$.

Whole-body VAT was estimated from VAT CSA with an algorithm from a healthy population ${ }^{(14)}$. Similar to skeletal muscle, predictive equations have not been developed for cancer populations; however, there is a strong relationship $(r 0.88)$ between VAT CSA at L3 and whole-body VAT volume in healthy humans ${ }^{(14)}$.

$$
\begin{aligned}
\text { VAT volume }(L)= & 0.0205 \times(\text { VAT CSA } 5 \mathrm{~cm} \text { above L4 } \\
& \left.-\mathrm{L} 5\left(\mathrm{~cm}^{2}\right)\right)+0.1475 ; r^{2} 0.945 .
\end{aligned}
$$

A density of $0.96 \mathrm{~g} / \mathrm{cm}^{3}$ was used to convert VAT volume into mass $(\mathrm{g})^{(19)}$.

There were patients who had portions of SAT that were not visible in the viewing field of the CT ( $n$ 22); for those patients for whom SAT could be viewed and calculated in its entirety ( $n$ 28), total adipose tissue (TAT) CSA was used to estimate whole-body adipose tissue. TAT was calculated as the sum of SAT, VAT and IMAT. Regression equations from a cohort of advanced cancer patients were used to estimate whole-body TAT from the surface area using the following equation ${ }^{(15)}$ :

$$
\begin{aligned}
\text { Whole-body fat mass }(\mathrm{kg})= & 0.042 \times\left(\mathrm{TAT} \text { at } \mathrm{L} 3\left(\mathrm{~cm}^{2}\right)\right) \\
& +11.2 ; r^{2} 0.77 .
\end{aligned}
$$

Net tissue change analysis for both skeletal muscle and VAT was based on the absolute difference between the first and last scans available for each patient (average 286 (sD 151) d; Fig. 1). Multiple scans were performed between the first and last scans for each patient; these scans were analysed and tissue change between consecutive scans was averaged over $100 \mathrm{~d}$ intervals from the time of death (i.e. $0-100 \mathrm{~d}$ before death, $101-200 \mathrm{~d}$ before death, etc.) to create a disease trajectory. The time intervals examined included approximately $70,150,250$ and $350 \mathrm{~d}$ from death. There were no significant differences in the rate of change or total tissue change between each consecutive time interval, thus net tissue changes were representative of the longitudinal changes during the disease trajectory (data not shown). Absolute changes in CSA were converted to mass using the aforementioned algorithms for skeletal muscle and VAT.

Absolute changes between the first and last scans were then calculated as a rate of change per $100 \mathrm{~d}$ to provide a more realistic time frame for assessing the rate of tissue loss. This also provided a standardised unit to allow comparisons between patients as the timing of CT images was unique for each individual.

$\Delta(\mathrm{kg}) / 100 \mathrm{~d}=(\mathrm{kg}$ at the last scan $-\mathrm{kg}$ at the first scan $)$

/total time survived $\times 100 \mathrm{~d}$.

\section{Statistical analysis}

Statistical analysis was performed using SigmaPlot ${ }^{\circledR}$ version 11.2 (Systat Software, Inc.). All values are presented as means and standard deviations, significance was identified at 
$P=0.05$ and trends were identified as $P=0 \cdot 120$. Power was set at 0.80 for all tests. Non-parametric statistics were performed on this dataset, as it was not normally distributed, while mathematical transformation could not correct the distribution. Comparisons between the two groups were performed by Mann-Whitney rank-sum tests and comparisons between multiple groups (tertile analysis) were performed by Kruskal-Wallis non-parametric one-way ANOVA with Dunn's method for multiple comparisons. Kaplan-Meier survival curves were constructed using the log-rank method for a comparison between multiple groups. The $\chi^{2}$ test was used to determine the relationships between cancer stage at diagnosis and secondary disease states (sarcopenia, diabetes and anaemia).

\section{Results}

\section{Patient characteristics}

A total of fifty pancreatic cancer patients met the study criteria. Patients were 66 (SD 12) years old with an equal distribution of males (56\%) and females (44\%). Average BMI was 23.4 $(\mathrm{sD} 3.7) \mathrm{kg} / \mathrm{m}^{2}$, and $58 \%$ of the patients were characterised as normal weight (BMI between 18.5 and $24.9 \mathrm{~kg} / \mathrm{m}^{2}$ ), as defined by the World Health Organization ${ }^{(20)}$. Despite normal BMI at diagnosis, $48 \%$ of the patients were characterised as sarcopenic. Only five of the patients were classified as underweight (BMI $<18.5 \mathrm{~kg} / \mathrm{m}^{2}$ ), indicating that sarcopenia was independent of overall body size (Table 1). Interestingly, sarcopenic patients were more likely to be diagnosed at an earlier stage (IIB) of disease compared with non-sarcopenic patients (stage IV, $P=0 \cdot 008$ ). However, cancer stage was not significantly related to the other clinical parameters assessed including presence of diabetes at diagnosis $(P=0.778)$, presence of anaemia at the time of the first CT scan $(P=0 \cdot 423)$, nutritional supplementation $(P=0 \cdot 219)$ or any other parameters listed in Table 1 . In terms of treatment, $62 \%$ of the patients underwent surgery at some point during the treatment trajectory, $46 \%$ received radiation and $88 \%$ received chemotherapy. The heterogeneity of this patient group allows for the identification of global changes in body composition associated with pancreatic cancer and for the identification of any clinical parameters that may be associated with increased muscle and adipose tissue loss. Physical characteristics of patients at diagnosis and treatment parameters are presented in Table 1.

\section{Net tissue change analysis}

Longitudinal analysis was performed to identify different rates of change in muscle and adipose tissue. There were no significant differences in total tissue change and rates of change between consecutive time intervals (i.e. approximately 70 , 150,250 and $350 \mathrm{~d}$ from death) throughout the disease trajectory for muscle or adipose tissue (data not shown). Absolute changes in muscle and VAT were representative of change throughout the disease trajectory; based on these findings, the remainder of the present results are based on net tissue changes. Overall, thirty-eight of the fifty patients (76.0\%) experienced net muscle loss, and $86.0 \%$ (forty-three out of the fifty patients) experienced net VAT loss over the disease trajectory. On average, approximately $1.72 \mathrm{~kg}(P<0.001)$ of

Table 1. Physical diagnostic characteristics and treatment parameters of patients $(n 50)$

(Mean values and standard deviations; number of patients, ranges and percentages)

\begin{tabular}{|c|c|c|}
\hline & $n$ & $\%$ \\
\hline \multicolumn{3}{|l|}{ Age (years) } \\
\hline Mean & \multicolumn{2}{|c|}{$66 \cdot 0$} \\
\hline SD & \multicolumn{2}{|c|}{$12 \cdot 0$} \\
\hline Range & \multicolumn{2}{|c|}{$49-84$} \\
\hline \multicolumn{3}{|l|}{ Weight (kg) } \\
\hline Mean & \multicolumn{2}{|c|}{$66 \cdot 0$} \\
\hline SD & \multicolumn{2}{|c|}{$12 \cdot 1$} \\
\hline \multicolumn{3}{|l|}{$\mathrm{BMI}\left(\mathrm{kg} / \mathrm{m}^{2}\right)$} \\
\hline Mean & \multirow{2}{*}{\multicolumn{2}{|c|}{$\begin{array}{c}23.4 \\
3.7\end{array}$}} \\
\hline SD & & \\
\hline Underweight & 5 & 10 \\
\hline Normal weight & 29 & 58 \\
\hline Overweight & 15 & 30 \\
\hline Obese & 1 & 2 \\
\hline \multicolumn{3}{|l|}{ Sex } \\
\hline Male & 28 & 56 \\
\hline Female & 22 & 44 \\
\hline Sarcopenic at diagnosis & 24 & 48 \\
\hline \multicolumn{3}{|l|}{ Male } \\
\hline No. of sarcopenic patients & 16 & 57 \\
\hline Average index $\left(\mathrm{cm}^{2} / \mathrm{m}^{2}\right)$ & 55.2 & $10 \cdot 0$ \\
\hline \multicolumn{3}{|l|}{ Female } \\
\hline No. of sarcopenic patients & 8 & 36 \\
\hline Average index $\left(\mathrm{cm}^{2} / \mathrm{m}^{2}\right)$ & $41 \cdot 2$ & $6 \cdot 3$ \\
\hline \multicolumn{3}{|l|}{ Diabetic at diagnosis } \\
\hline Yes & 18 & 36 \\
\hline No & 32 & 64 \\
\hline \multicolumn{3}{|l|}{ Anaemic at first CT scan } \\
\hline Yes & 24 & 48 \\
\hline No & 26 & 52 \\
\hline \multicolumn{3}{|l|}{ Tumour stage } \\
\hline IIB & 15 & 30 \\
\hline III & 18 & 36 \\
\hline IV & 16 & 32 \\
\hline Unknown & 1 & 2 \\
\hline \multicolumn{3}{|l|}{ Histology } \\
\hline Adenocarcinoma & 49 & 98 \\
\hline Carcinoma & 1 & 2 \\
\hline \multicolumn{3}{|l|}{ Surgery* } \\
\hline Yes & 31 & 62 \\
\hline No & 19 & 38 \\
\hline \multicolumn{3}{|l|}{ Radiation therapy } \\
\hline Yes & 23 & 46 \\
\hline No & 27 & 54 \\
\hline \multicolumn{3}{|l|}{ Chemotherapy† } \\
\hline Chemotherapy & 44 & 88 \\
\hline No chemotherapy & 6 & 12 \\
\hline \multicolumn{3}{|c|}{ No. of patients receiving nutritional consultation } \\
\hline Yes & 32 & 64 \\
\hline No & 18 & 36 \\
\hline \multicolumn{3}{|c|}{ Number of patients receiving nutritional supplementationł } \\
\hline Yes & 27 & 54 \\
\hline No & 23 & 46 \\
\hline
\end{tabular}

$\mathrm{CT}$, computed tomography.

* Surgery mainly included radical $(78 \%)$, palliative or bypass surgeries, and endoscopic retrograde cholangiopancreatography (ERCP) with stent insertion.

$\uparrow$ Chemotherapy regimens predominantly included gemcitabine (89\%), 5-flourouricil (infusion or bolus) and capecitabine.

‡ Nutritional supplementations included Ensure, Carnation Breakfast Anytime and protein powder. 
muscle was lost at a rate of $-0.55(\mathrm{SD} 1.00) \mathrm{kg} / 100 \mathrm{~d}$ $(P<0.001 ;$ Table 2). Patients also lost approximately $1.04 \mathrm{~kg}$ of VAT $(P<0.001)$ between the first and last CT images at a rate of -0.32 (SD 0.39) kg/100d ( $P<0.001$; Table 2). Tissue losses were then ranked based on total loss over the disease trajectory and were subsequently divided into equal tertiles of approximately seventeen patients per group. Patients in tertile 1 lost 4.93 (SD 2.50) kg of muscle, while patients in tertile 3 actually gained a small amount of muscle (1.18 $(\mathrm{SD} 1.31) \mathrm{kg}$ ) over the disease trajectory $(P<0 \cdot 001$; Table 3$)$. VAT showed a similar trend with patients in tertile 1 losing $2 \cdot 24$ (SD 0.67$) \mathrm{kg}$ of VAT and patients in tertile 3 showing a relatively little change in VAT $(0.04(\mathrm{SD} 0.48) \mathrm{kg})(P<0.001$; Table 3$)$. The rate of change in muscle and VAT followed the same distribution as absolute tissue loss $(P<0 \cdot 001$; Table 3$)$.

Interestingly, patients who lost VAT at the most accelerated rate (average $-0.74(\mathrm{sD} 0.36) \mathrm{kg} / 100 \mathrm{~d}$, tertile 1$)$ demonstrated shorter average survival time (316.8 (SD 26.3) d) compared with patients who lost VAT at the slowest rate $(515 \cdot 1$ (SD 62.9) d, average $-0.02(\mathrm{SD} 0.07) \mathrm{kg} / 100 \mathrm{~d}$, tertile 3), but not patients with moderate VAT loss (423.8 (SD 55.5) d, average -0.25 (sD 0.09) kg/100 d, $P=0.020$; Fig. 2(a)). Also, patients who lost muscle at the most accelerated rate (average -1.59 (SD 1.00$) \mathrm{kg} / 100 \mathrm{~d}$, tertile 1) tended to demonstrate shorter survival time (333.0 (SD 38.7) d) compared with patients who lost muscle at slower rates $(508.4$ (SD 57.3) d, average -0.32 (SD $0 \cdot 19) \mathrm{kg} / 100 \mathrm{~d}$, tertile 2) and those who gained muscle (413.8 (sD 56.8) d, average $0.29($ sD 0.32$) \mathrm{kg} / 100 \mathrm{~d}$, tertile 3, $P=0 \cdot 104$; Fig. 2(b)). However, there was no significant difference in survival time between patients who lost the most VAT or skeletal muscle compared with patients who lost smaller absolute amounts of tissue (data not shown, $P=0.876$ and $P=0.884$, respectively). This suggests that the rate of tissue loss, not the absolute amount of tissue loss, may be specifically important in patient survival.

Patients also lost significant amounts of IMAT, SAT and TAT during the course of the disease trajectory. Patients lost $-3.85 \mathrm{~cm}^{2}$ of IMAT at a rate of -12.0 (SD 17.5) $\% / 100 \mathrm{~d}$ $(P<0.001)$. A subset of twenty-eight patients was used to quantify changes in SAT and TAT, as SAT was cut off from the field of view in twenty-two out of the fifty patients. With the exception of this latter subset having less SAT mass, they were similar to the former subgroup, as there were no differences in any of the descriptive characteristics (data not shown). Patients lost 4.82 (SD 4.44) kg of TAT at a rate of $-1.53(\mathrm{SD} 1.65) \mathrm{kg} / 100 \mathrm{~d}$ over the disease trajectory.
TAT loss appears to be accelerated when compared with VAT loss (0.32 (SD 0.39) kg/100 d v. - 1.53 (SD 1.65) kg/100 d); however, TAT accounts for 43.7 (SD 16.7) \% of total mass at diagnosis, while VAT accounts for only 3.58 (SD 2.02)\%. When looking at a percentage change per $100 \mathrm{~d}$ in these tissues, we see that there was no significant difference in the rate of loss for VAT, SAT, IMAT and TAT. VAT was lost at a rate of $-15.5(\mathrm{SD} 17.9) \% / 100 \mathrm{~d}$, SAT at a rate of -13.5 (SD $14 \cdot 1) \% / 100 \mathrm{~d}$, IMAT at a rate of $-12 \cdot 0(\mathrm{SD} 17 \cdot 5) \% / 100 \mathrm{~d}$ and TAT at a rate of $-14.4(\mathrm{SD} 13 \cdot 2) \% / 100 \mathrm{~d}(P=0.384)$. These data suggest that changes observed in VAT were similar to the loss of other adipose tissue depots.

\section{Determinants of visceral adipose tissue loss}

Physical and clinical characteristics were used to assess determinants of VAT loss. As the rate of VAT loss appears to predict survival, the amount of VAT lost between patients surviving for $>1$ year and patients surviving $<1$ year was examined. Patients surviving for $>1$ year lost the same amount of VAT as patients who survived for $<1$ year $(-1.03$ (SD 1.11) kg $v$. -1.05 (SD 1.08) kg, $P=0.755$; Table 4); however, patients surviving for $<1$ year lost VAT at faster rates than those who survived for $>1$ year $(-0.45$ (SD 0.48$) \mathrm{kg} / 100 \mathrm{~d} v$. -0.22 (sD 0.25$) \mathrm{kg} / 100 \mathrm{~d}, P=0.041$; Table 4). Again, this supports the notion that the rate of VAT loss may be more important in survival than the total amount of tissue lost.

Non-sarcopenic patients tended to lose VAT at an accelerated rate when compared with sarcopenic patients $(-0.35$ (SD 0.32$) \mathrm{kg} / 100 \mathrm{~d} v$. -0.26 (SD 46) $\mathrm{kg} / 100 \mathrm{~d}$, respectively, $P=0 \cdot 115$; Table 4); however, there were no significant differences in total VAT loss between non-sarcopenic and sarcopenic patients $(-1.21$ (SD 1.03$) \mathrm{kg} v$. -0.77 (SD 1.13) kg, respectively, $P=0 \cdot 161$; Table 4$)$.

Also, males tended to lose more VAT and at an accelerated rate, when compared with females $(-1.27$ (SD 1.16) kg $v$. -0.75 (sD 0.92) kg, $P=0.092$, respectively; $-0.42(\mathrm{sD} 0.43) \mathrm{kg} /$ $100 \mathrm{~d} v . \quad-0.19(\mathrm{SD} 0 \cdot 29) \mathrm{kg} / 100 \mathrm{~d}, P=0.068$, respectively; Table 4). To further investigate this discrepancy between males and females, we examined the amount of VAT lost as a proportion of total body weight at diagnosis. In this case, males presented with greater body weight at diagnosis compared with females $(70.1$ (SD 8.71) kg $v$. 60.7 (SD 13.9) kg, respectively), thus a greater VAT loss was proportional to the larger body weight in males. When examining the data from this respect, there were no significant differences between

Table 2. Tissue characteristics

(Mean values and standard deviations)

\begin{tabular}{|c|c|c|c|c|c|c|c|c|}
\hline & \multicolumn{2}{|c|}{ First scan } & \multicolumn{2}{|c|}{ Last scan } & \multicolumn{2}{|c|}{$\Delta$} & \multicolumn{2}{|c|}{$\Delta / 100 \mathrm{~d}$} \\
\hline & Mean & SD & Mean & SD & Mean & SD & Mean & SD \\
\hline Muscle $\left(\mathrm{cm}^{2}\right)$ & $129 \cdot 30$ & $3 \cdot 25$ & $119 \cdot 30^{*}$ & $27 \cdot 10$ & -9.96 & $17 \cdot 50$ & $-3 \cdot 21$ & $5 \cdot 77$ \\
\hline Muscle (kg) & 24.50 & 5.60 & $22 \cdot 80^{*}$ & 4.69 & -1.72 & 3.29 & -0.55 & 1.00 \\
\hline VAT $\left(\mathrm{cm}^{2}\right)^{\prime \prime}$ & $111 \cdot 10$ & $73 \cdot 10$ & $58 \cdot 40^{*}$ & $56 \cdot 30$ & -52.80 & 55.00 & $-16 \cdot 40$ & $19 \cdot 70$ \\
\hline VAT $(\mathrm{kg})$ & 2.33 & 1.44 & $1.29^{\star}$ & $1 \cdot 11$ & -1.04 & 1.08 & -0.32 & 0.39 \\
\hline
\end{tabular}

VAT, visceral adipose tissue.

${ }^{\star}$ Mean values were significantly different between the first and last scans $(P<0.05)$. 
Table 3. Tertile analysis

(Mean values and standard deviations)

\begin{tabular}{|c|c|c|c|c|c|c|}
\hline & \multicolumn{2}{|c|}{ Tertile 1} & \multicolumn{2}{|c|}{ Tertile 2} & \multicolumn{2}{|c|}{ Tertile 3} \\
\hline & Mean & SD & Mean & SD & Mean & SD \\
\hline Muscle (kg) & -4.93 & 2.50 & $-1.23^{*}$ & 0.64 & $1.18^{*}$ & 1.31 \\
\hline Rate of muscle change $(\mathrm{kg} / 100 \mathrm{~d})$ & -1.59 & 1.00 & $-0.32^{\star}$ & 0.19 & $0.29^{*}$ & 0.32 \\
\hline VAT $(\mathrm{kg})$ & $-2 \cdot 24$ & 0.67 & $-0.90^{\star}$ & 0.36 & $0.04^{*}$ & 0.48 \\
\hline Rate of VAT change $(\mathrm{kg} / 100 \mathrm{~d})$ & -0.74 & 0.36 & $-0.25^{\star}$ & 0.09 & $-0.02^{*}$ & 0.07 \\
\hline
\end{tabular}

VAT, visceral adipose tissue.

${ }^{*}$ Mean values were significantly different from those of tertile $1(P<0.05)$.

males and females in the proportion of VAT lost relative to body weight $(-1.77$ (SD 1.64)\% v. -1.32 (SD 1.75$) \%$, $P=0.333$ ). In addition, there were no significant differences observed in the amount of VAT lost or the rate of VAT loss relative to age or BMI (Table 4). Chemotherapy was not assessed as a clinical parameter, as $88 \%$ of the patients received chemotherapy at some point during the disease trajectory.

Patients presenting with diabetes at diagnosis lost significantly more VAT when compared with patients who were not diabetic at diagnosis $(-1.50$ (SD 0.99) kg $v$. -0.74 (sD 1.06) kg, $P=0.017$; Table 4). Diabetic patients also lost VAT at an accelerated rate when compared with non-diabetic patients ( $-0.44($ SD 0.33$) \mathrm{kg} / 100 \mathrm{~d} v .-0.20($ SD 0.30$) \mathrm{kg} / 100 \mathrm{~d}$, $P=0 \cdot 010$; Table 4). This also corresponded to a greater proportion of VAT relative to total body weight lost by diabetic patients compared with non-diabetic patients ( $-2 \cdot 14$ (SD $1.49) \% v$. -1.17 (SD 1.65) \%, respectively, $P=0.033$ ). There were no significant differences in absolute VAT loss and the rate of VAT loss for any of the other clinical parameter listed in Table 4. A description of the characteristics of the subpopulations examined can be found in Table 5 .

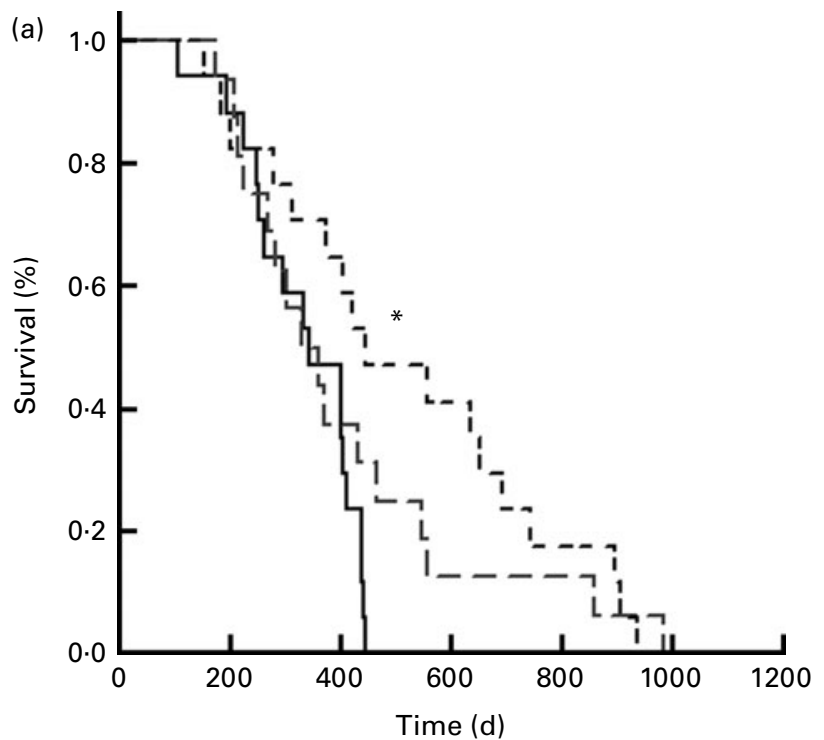

\section{Determinants of skeletal muscle loss}

Muscle tissue was lost at accelerated rates when compared with an ageing population followed over 12 years (age 65.4 (SD 4.2) years at the first evaluation), who typically lose muscle at a rate of approximately $1.4 \%$ per year ${ }^{(17)}$, and cross-sectional studies have demonstrated a loss of approximately $1 \%$ of muscle per year (age range $15-83$ years) ${ }^{(18)}$. In the present study, muscle CSA was lost at a rate of approximately $7 \cdot 48 \%$ per year.

Also, patients who were anaemic at the first CT scan lost more muscle and at an accelerated rate when compared with non-anaemic patients $(-2.41$ (SD 3.17) kg $v$. -1.00 (sD 2.78$) \mathrm{kg}$, respectively, $P=0.029$; -0.71 (SD 0.87) kg/100 d v. $-0.29(\mathrm{sD} 0.85) \mathrm{kg} / 100 \mathrm{~d}$, respectively, $P=0.017$; Table 4). Anaemic patients also lost a greater proportion of total initial body weight compared with non-anaemic patients $(-3.58$ (SD 4.76)\% $v .-1.07$ (SD 3.15) \%, respectively, $P=0.018$ ). The presence of diabetes at diagnosis tended to influence the amount of muscle lost, with diabetic patients tending to lose more muscle than non-diabetic patients $(-2.58$ (SD 4.01$) \mathrm{kg}$ v. $-1 \cdot 14(\mathrm{SD} 2 \cdot 18) \mathrm{kg}$, respectively, $P=0 \cdot 110$; Table 4).

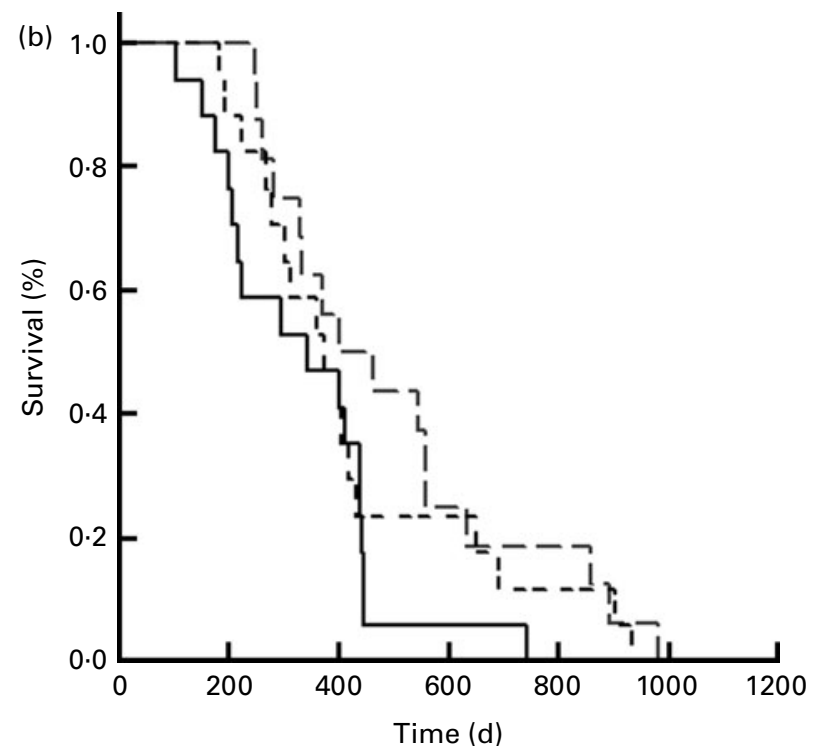

Fig. 2. Kaplan-Meier survival curves: division of patients based on tertiles of the rate of tissue loss in $\mathrm{kg} / 100 \mathrm{~d}$. (a) Rate of change in visceral adipose tissue (VAT). *Values were significantly different between tertile 1 ( -$)$ and tertile $3(\ldots .).(P<0.05)$. Tertile 1 : VAT loss $>-0.40 \mathrm{~kg} / 100 \mathrm{~d}$; tertile $2(--$ ): VAT loss $<-0.40$ and $>-0.10 \mathrm{~kg} / 100 \mathrm{~d}$; tertile 3: VAT loss $<-0.10 \mathrm{~kg} / 100 \mathrm{~d}$. (b) Rate of change in skeletal muscle; tertile 1 : rate of muscle loss $>-0.70 \mathrm{~kg} / 100 \mathrm{~d}$; tertile 2: rate of muscle loss $<-0.70$ and $<-0.10 \mathrm{~kg} / 100 \mathrm{~d}$; tertile 3 : rate of muscle loss $<-0.10 \mathrm{~kg} / 100 \mathrm{~d}$. 
Table 4. Influence of physical diagnostic and clinical characteristics on tissue changes

(Mean values and standard deviations)

\begin{tabular}{|c|c|c|c|c|c|c|c|c|c|}
\hline \multirow[b]{3}{*}{ Characteristics } & \multirow[b]{3}{*}{$n$} & \multicolumn{4}{|c|}{ VAT } & \multicolumn{4}{|c|}{ Muscle } \\
\hline & & \multicolumn{2}{|c|}{$\Delta(\mathrm{kg})$} & \multicolumn{2}{|c|}{$\Delta(\mathrm{kg} / 100 \mathrm{~d})$} & \multicolumn{2}{|c|}{$\Delta(\mathrm{kg})$} & \multicolumn{2}{|c|}{$\Delta(\mathrm{kg} / 100 \mathrm{~d})$} \\
\hline & & Mean & SD & Mean & SD & Mean & SD & Mean & SD \\
\hline \multicolumn{10}{|l|}{ Survival } \\
\hline$>1$ year & 27 & -1.03 & $1 \cdot 11$ & $-0.22^{*}$ & 0.25 & -1.81 & 3.34 & -0.36 & 0.76 \\
\hline$<1$ year & 23 & -1.05 & 1.08 & -0.45 & 0.48 & -1.62 & $2 \cdot 66$ & -0.78 & $1 \cdot 20$ \\
\hline \multicolumn{10}{|l|}{ Sex } \\
\hline Male & 28 & -1.27 & $1 \cdot 16$ & -0.42 & 0.43 & -2.47 & 3.48 & -0.84 & 1.17 \\
\hline Female & 22 & -0.75 & 0.92 & -0.19 & 0.29 & -0.76 & 1.97 & -0.19 & 0.55 \\
\hline \multicolumn{10}{|l|}{ Sarcopenia present } \\
\hline Yes & 24 & -0.77 & $1 \cdot 13$ & -0.26 & 0.46 & -0.93 & 3.09 & $-0.39^{\star}$ & $1 \cdot 18$ \\
\hline No & 26 & $-1 \cdot 21$ & 1.03 & -0.35 & 0.32 & $-2 \cdot 26$ & $2 \cdot 86$ & -0.65 & 0.80 \\
\hline \multicolumn{10}{|l|}{ BMI } \\
\hline$>25 \mathrm{~kg} / \mathrm{m}^{2}$ & 14 & $-1 \cdot 34$ & 1.09 & -0.38 & 0.35 & $-2 \cdot 85$ & 3.52 & -0.67 & 0.87 \\
\hline$<25 \mathrm{~kg} / \mathrm{m}^{2}$ & 35 & -0.90 & 1.08 & -0.29 & 0.40 & $-1 \cdot 21$ & $2 \cdot 73$ & -0.49 & 1.06 \\
\hline \multicolumn{10}{|l|}{ Age } \\
\hline$>65$ years old & 26 & -0.98 & $1 \cdot 18$ & -0.26 & 0.33 & -1.63 & 2.65 & -0.52 & 0.83 \\
\hline$<65$ years old & 24 & $-1 \cdot 10$ & 0.98 & -0.39 & 0.44 & -1.82 & 3.42 & -0.59 & $1 \cdot 16$ \\
\hline \multicolumn{10}{|l|}{ Stage } \\
\hline IIB & 15 & $-1 \cdot 14$ & 1.07 & -0.30 & 0.34 & -0.30 & $2 \cdot 31$ & -0.05 & 0.50 \\
\hline III & 18 & -0.87 & $1 \cdot 11$ & -0.25 & 0.30 & -2.07 & 2.77 & -0.59 & 0.89 \\
\hline IV & 16 & -1.03 & $1 \cdot 13$ & -0.33 & 0.33 & -2.56 & 3.56 & -0.80 & 1.00 \\
\hline \multicolumn{10}{|l|}{ Diabetes present } \\
\hline Yes & 18 & $-1.50^{\star}$ & 0.99 & $-0.44^{\star}$ & 0.33 & -2.58 & 4.01 & -0.70 & 1.07 \\
\hline No & 31 & -0.74 & 1.06 & -0.20 & 0.30 & -1.14 & $2 \cdot 18$ & -0.35 & 0.70 \\
\hline \multicolumn{10}{|l|}{ Anaemia present } \\
\hline Yes & 24 & -0.99 & $1 \cdot 11$ & -0.28 & 0.30 & $-2 \cdot 41^{*}$ & 3.17 & $-0.71^{*}$ & 0.87 \\
\hline No & 25 & -1.04 & $1 \cdot 10$ & -0.30 & 0.36 & -1.00 & $2 \cdot 78$ & -0.29 & 0.85 \\
\hline \multicolumn{10}{|l|}{ Surgery } \\
\hline Yes & 31 & $-1 \cdot 18$ & $1 \cdot 14$ & -0.31 & 0.33 & -2.08 & 3.50 & -0.49 & 0.90 \\
\hline No & 18 & -0.73 & 0.96 & -0.25 & 0.33 & -1.02 & 1.90 & -0.50 & 0.86 \\
\hline \multicolumn{10}{|l|}{ Radiation therapy } \\
\hline Yes & 23 & -1.06 & $1 \cdot 11$ & -0.28 & 0.26 & -1.27 & $2 \cdot 50$ & -0.40 & 0.83 \\
\hline No & 26 & -0.97 & 1.09 & -0.30 & 0.37 & -2.06 & 3.44 & -0.57 & 0.92 \\
\hline \multicolumn{10}{|l|}{ Dietetic consult } \\
\hline Yes & 32 & -0.95 & $1 \cdot 11$ & -0.26 & 0.34 & -1.44 & 3.26 & -0.38 & 0.89 \\
\hline No & 17 & $-1 \cdot 19$ & 1.04 & -0.43 & 0.46 & $-2 \cdot 23$ & 2.53 & -0.86 & $1 \cdot 13$ \\
\hline \multicolumn{10}{|l|}{ Supplementation } \\
\hline Yes & 27 & -0.85 & 1.03 & -0.23 & 0.32 & $-0.78^{*}$ & 2.44 & $-0.19^{*}$ & 0.65 \\
\hline No & 23 & -1.44 & $1 \cdot 13$ & -0.50 & 0.46 & $-3 \cdot 26$ & 3.39 & $-1 \cdot 16$ & 1.25 \\
\hline
\end{tabular}

VAT, visceral adipose tissue.

${ }^{*}$ Mean values were significantly different between the two groups compared $(P<0.05)$.

Also, patients who received nutritional supplementation lost significantly less skeletal muscle and the rate of loss was significantly attenuated when compared with patients who did not receive nutritional supplementation $(-0.78$ (SD 2.44) kg $v$. -3.26 (SD 3.39) kg, respectively, $P=0.026$; $-0.19(\mathrm{sD} \quad 0.65) \mathrm{kg} / 100 \mathrm{~d} \quad v \cdot-1.16 \quad(\mathrm{sD} \quad 1.25) \mathrm{kg} / 100 \mathrm{~d}$, respectively, $P=0.009$; Table 4 ). Patients receiving nutritional supplementation lost a similar proportion of initial body weight compared with patients not receiving nutritional supplementation ( -1.77 (SD 4.58) v. $-3 \cdot 10$ (SD 3.60), $P=0 \cdot 199)$; however, patients receiving supplementation had a significantly lower initial body weight $(62 \cdot 8$ (SD 10.3) kg) compared with patients not receiving supplementation (69.8 (SD 13.3$) \mathrm{kg}, P=0.041$ ), which may influence this finding. When examining survival time for patients receiving nutritional supplementation using Kaplan-Meier survival curves, patients who received nutritional supplementation tended to live longer than patients who did not receive nutritional supplementation (median: 468.2 (SD 46.2) d $\quad v$. $360 \cdot 2$ (SD 37.0) d, respectively, $P=0.098$, curves not shown). Nutritional supplementation was associated with dietetic consult $(P<0 \cdot 001)$; however, dietetic consult alone was not associated with changes in muscle $(P=0.395 ;$ Table 4$)$. There were no significant differences in muscle loss for any of the other characteristics examined (Table 4).

Similar to VAT, males compared with females tended to lose more muscle tissue and at an accelerated rate $(-2.47$ (SD 3.48) kg $v .-0.76($ sD 1.97$) \mathrm{kg}, P=0.084 ;-0.84($ sD 1.17$) \mathrm{kg} /$ $100 \mathrm{~d} v$. $-0.19(\mathrm{sD} 0.55) \mathrm{kg} / 100 \mathrm{~d}, P=0.065$; Table 4). However, there were no significant differences between the proportion of muscle mass relative to total body weight between males and females (-3.24 (SD 4.54)\% v. $-1 \cdot 19$ (SD $3 \cdot 46) \%, P=0 \cdot 151$ ). Also, patients classified as sarcopenic at the time of the first scan tended to lose less muscle when compared with patients classified as non-sarcopenic at their first scan $(-0.93$ (SD 3.09) kg v. -2.26 (sD 2.86) kg, 
Table 5. Characteristics of the subpopulations analysed $\dagger$

\begin{tabular}{|c|c|c|c|c|c|c|c|c|c|c|c|c|c|c|c|}
\hline \multirow[b]{2}{*}{ Characteristics } & \multirow[b]{2}{*}{$n$} & \multicolumn{2}{|c|}{ Sarcopenia } & \multicolumn{2}{|c|}{ Diabetes } & \multicolumn{2}{|c|}{ Anaemia } & \multicolumn{2}{|c|}{ Surgery } & \multicolumn{2}{|c|}{ Radiation } & \multicolumn{2}{|c|}{$\begin{array}{l}\text { Dietetic } \\
\text { consult }\end{array}$} & \multicolumn{2}{|c|}{$\begin{array}{l}\text { Supple- } \\
\text { mentation }\end{array}$} \\
\hline & & Yes & No & Yes & No & Yes & No & Yes & No & Yes & No & Yes & No & Yes & No \\
\hline \multicolumn{16}{|l|}{ Sex } \\
\hline Male & 28 & 15 & 13 & 11 & 16 & 17 & 10 & 16 & 11 & 10 & 17 & 16 & 11 & 13 & 15 \\
\hline Female & 22 & 8 & 13 & 7 & 15 & 7 & 15 & 15 & 7 & 13 & 9 & 16 & 6 & 14 & 8 \\
\hline Average BMI $\left(\mathrm{kg} / \mathrm{m}^{2}\right)$ & 50 & 23.0 & 23.7 & 24.9 & $22 \cdot 6$ & 23.8 & 23.0 & 23.6 & $23 \cdot 1$ & $22 \cdot 6$ & 24.13 & $22 \cdot 9$ & $24 \cdot 6$ & $22 \cdot 5$ & 24.4 \\
\hline Average age (years) & 50 & 65.5 & $63 \cdot 2$ & $64 \cdot 3$ & $64 \cdot 3$ & 63.7 & $64 \cdot 8$ & 63.4 & $66 \cdot 2$ & 63.3 & 65.5 & $64 \cdot 1$ & $64 \cdot 6$ & $64 \cdot 8$ & 63.9 \\
\hline \multicolumn{16}{|l|}{ Stage } \\
\hline IIB & 15 & 10 & 5 & 5 & 10 & 6 & 9 & 15 & $0^{*}$ & 8 & 7 & 12 & 3 & 11 & 4 \\
\hline III & 18 & 10 & 8 & 6 & 12 & 11 & 7 & 7 & 11 & 9 & 9 & 11 & 7 & 9 & 9 \\
\hline IV & 16 & $2^{*}$ & 13 & 7 & 9 & 7 & 9 & 9 & 7 & 6 & 10 & 9 & 7 & 7 & 9 \\
\hline \multicolumn{16}{|l|}{ Sarcopenia } \\
\hline Yes & 24 & - & - & 6 & 16 & 10 & 12 & 16 & 16 & 10 & 12 & 16 & 6 & 15 & 8 \\
\hline No & 26 & - & - & 11 & 15 & 14 & 12 & 14 & 12 & 12 & 14 & 16 & 10 & 11 & 15 \\
\hline \multicolumn{16}{|l|}{ Diabetes } \\
\hline Yes & 18 & - & - & - & - & 6 & 12 & 10 & 8 & 7 & 11 & 14 & 4 & 12 & 6 \\
\hline No & 31 & - & - & - & - & 18 & 13 & 21 & 10 & 16 & 15 & 18 & 13 & 15 & 16 \\
\hline \multicolumn{16}{|l|}{ Anaemia } \\
\hline Yes & 24 & - & - & - & - & - & - & 15 & 9 & 11 & 13 & 14 & 10 & 10 & 14 \\
\hline No & 25 & - & - & - & - & - & - & 16 & 9 & 12 & 13 & 18 & 7 & 17 & 8 \\
\hline \multicolumn{16}{|l|}{ Surgery } \\
\hline Yes & 31 & - & - & - & - & - & - & - & - & 16 & 15 & 20 & 12 & 17 & 14 \\
\hline No & 18 & - & - & - & - & - & - & - & - & 7 & 11 & 11 & 6 & 10 & 8 \\
\hline \multicolumn{16}{|l|}{ Radiation therapy } \\
\hline Yes & 23 & - & - & - & - & - & - & - & - & - & - & 17 & 6 & 17 & 6 \\
\hline No & 26 & - & - & - & - & - & - & - & - & - & - & 15 & 11 & 10 & 16 \\
\hline \multicolumn{16}{|l|}{ Dietetic consult } \\
\hline Yes & 32 & - & - & - & - & - & - & - & - & - & - & - & - & 25 & 7 \\
\hline No & 17 & - & - & - & - & - & - & - & - & - & - & - & - & $2^{*}$ & 15 \\
\hline
\end{tabular}

${ }^{*}$ Values were significantly different between the expected proportion of patients in the subpopulation and the actual proportion of patients in the subpopulation $(P<0.05)$. $\dagger$ Values represent the number of patients within each subpopulation (columns) with each characteristic examined (rows), except for average BMI and average age.

respectively, $P=0 \cdot 054$; Table 4$)$. Non-sarcopenic patients lost muscle at an accelerated rate when compared with sarcopenic patients $(-0.65(\mathrm{SD} 0 \cdot 80) \mathrm{kg} / 100 \mathrm{~d} v .-0.39(\mathrm{SD} 1 \cdot 18) \mathrm{kg} / 100 \mathrm{~d}$, respectively, $P=0.025$; Table 4$)$. Sarcopenic patients also tended to live longer than non-sarcopenic patients $(456.3$ (SD 55.7) d $v \cdot 418.8$ (SD 64.5) d, $P=0.051$ ), which may be explained by the finding that sarcopenic patients were diagnosed with earlier-stage cancer compared with nonsarcopenic patients. There were no significant influences of any other physical characteristics on changes in skeletal muscle (Table 4). A description of the characteristics of the subpopulations examined can be found in Table 5 . CA19-9 was evaluated in the twenty-eight patients with available data and was not related to changes in body composition (data not shown). Multivariate linear regression was performed to evaluate the potential interactions between each parameter. There were no significant interactions identified.

\section{Discussion}

The major finding of the present study is that the rate at which pancreatic cancer patients lose VAT and skeletal muscle provides an important indicator of survival compared with the total amount of tissue lost. Despite patients losing similar amounts of VAT over the disease trajectory, patients who survived for $>1$ year had attenuated rates of VAT loss compared with patients who survived for $<1$ year. We demonstrated that muscle tissue was lost at accelerated rates (approximately $7 \cdot 48 \%$ per year) in this group of cancer patients, which is far more exacerbated than aged individuals (15-83 years old) who typically lose muscle at a rate of $1-1.4 \%$ per year $^{(21,22)}$.

Additionally, this is the first study to identify that the presence of diabetes at diagnosis was associated with significantly more VAT loss and a tendency for greater muscle loss in pancreatic cancer patients. The presence of anaemia at the first CT scan was also associated with significant and accelerated losses of muscle compared with patients with normal $\mathrm{Hb}$ levels. These data suggest that anaemic and diabetic patients may be at a greater risk of cachexia when diagnosed with pancreatic cancer. The presence of diabetes at diagnosis or anaemia at the first CT scan, both contributors of muscle wasting and cachexia ${ }^{(6)}$, may serve as potential biomarkers of patients most susceptible to cancer cachexia. We demonstrated that use of nutritional supplementation may decrease the amount of skeletal muscle lost and attenuate the rate of tissue loss, which may improve survival. The present study demonstrated that patients receiving nutritional supplementation tended to live longer than patients not receiving supplementation, which warrants future investigation into this hypothesis. Identification of potential predictors of cachexia may allow early detection in patients who are at a risk of developing cachexia, which may permit the development of interventions near the time of diagnosis of pancreatic cancer patients. 
Rate of tissue loss may provide a better indicator of survival than total tissue lost

Pancreatic cancer patients have the highest incidence rates of cancer cachexia and experience the most severe symptoms of cachexia $^{(4)}$. While few studies have characterised the specific changes in muscle and adipose tissue, we demonstrated that pancreatic cancer patients lose both muscle and fat during the disease trajectory. The patterns of tissue loss in the present study are different from those observed in other cancer populations such as colorectal cancer ${ }^{(23)}$; thus, each cancer type should be considered separately. Despite its metabolic importance, changes in VAT quantitatively represent changes in all adipose tissue depots, as demonstrated by Hallgreen \& Hall $^{(24)}$ and confirmed in the present study. In a recent study by Tan et al. ${ }^{(8)}$, forty-four pancreatic cancer patients lost $-2 \cdot 1$ (SD $4 \cdot 1) \mathrm{kg}$ of lean mass and -4.5 (SD 3.7) $\mathrm{kg}$ of TAT mass during the $189 \mathrm{~d}$ disease trajectory. The patients in the present study were similar to the Tan et al. ${ }^{(8)}$ patient cohort in terms of age, BMI and sex, but the patients in the present study survived longer. Thus, when patients were grouped by those surviving $<1$ year $v$. those surviving $>1$ year, we observed that all pancreatic patients lost similar amounts of total muscle and adipose tissue. However, the rate at which muscle and adipose tissue was lost influenced survival. Patients who lost VAT at the most accelerated rates had the poorest survival outcomes and patients who lost muscle at the fastest rate also tended to have the poorest survival outcomes. Patients with the most accelerated tissue losses may exhibit increased stimulation of the catabolic pathways associated with muscle and adipose wasting, such as increased pro-inflammatory processes, and inhibition of the anabolic pathways ${ }^{(25,26)}$. Attenuating the rate of muscle and adipose tissue loss through these pathways may improve survival in pancreatic cancer patients.

\section{Determinants of visceral adipose tissue and muscle loss are independent and unique}

Pancreatic cancer patients who had been diagnosed with diabetes before their cancer diagnosis lost significantly more VAT and tended to lose more skeletal muscle when compared with non-diabetic patients. The development of glucose intolerance has been documented in advanced cancer patients with cachexia ${ }^{(27)}$, and is a defining characteristic of diabetes. Pancreatic cancer patients with impaired glucose handling at diagnosis may be predisposed to changes in body composition, as their pre-existing glucose intolerance may exacerbate changes in muscle and adipose tissue during the disease trajectory. Patients who are identified as diabetic at cancer diagnosis are exposed to perturbed glucose and insulin metabolism for a longer duration of time compared with patients who develop diabetes during the disease trajectory. Patients with longer-term diabetes (i.e. diabetic at cancer diagnosis) may have accelerated losses of muscle and adipose tissue for this reason.

The association between diabetes and tissue loss has also been demonstrated in elderly populations with type 2 diabetes $^{(28,29)}$. In these studies, elderly individuals with type 2 diabetes display a greater magnitude of sarcopenia, compared with elderly individuals without type 2 diabetes. Also, Wang et al. ${ }^{(5)}$ demonstrated that insulin resistance accelerates muscle protein degradation in $d b / d b$ mice via the ubiquitin-proteasome pathway, the pathway most often implicated in muscle wasting in cancer cachexia ${ }^{(30)}$. Patients presenting with impaired glucose handling at diagnosis may be at an increased risk of muscle and adipose tissue loss.

The presence of anaemia at the first CT scan also significantly influenced the rate of skeletal muscle loss, with anaemic patients losing significantly more muscle at an accelerated rate when compared with non-anaemic patients. The development of anaemia in conjunction with cachexia has been documented and the presence of anaemia is one of the biochemical markers used to diagnose cachexia ${ }^{(6)}$. This suggests that patients who are anaemic at the first CT scan may be at an increased risk of weight loss and may have poorer survival outcomes. Anaemia affects the ability of erythrocytes to efficiently deliver $\mathrm{O}_{2}$ to peripheral tissues. Impaired $\mathrm{O}_{2}$ delivery may therefore exacerbate the rate of muscle loss by impairing the tissue's ability to oxidise metabolic substrates. Anaemia in chronic disease states is associated with increased inflammation ${ }^{(31)}$, which, in some diseases such as chronic kidney disease, has been linked to muscle wasting ${ }^{(32)}$. It is possible that inflammation may also be mediating the influence of anaemia on muscle wasting in pancreatic cancer patients. Altogether, the interrelationship between diabetes, anaemia and muscle loss suggests that availability of glucose and inflammation may influence the rate of muscle loss, ultimately contributing to cachexia.

Patients who received nutritional supplementation lost significantly less muscle at a slower rate when compared with patients who did not receive any type of nutritional supplementation. Supplemented patients also tended to live longer than non-supplemented patients. This was independent of the stage of cancer at diagnosis. The nutritional supplements varied across patients and included protein powder, Ensure, Boost, Carnation Breakfast Anytime, Glucerna, vitamin and mineral supplementations. Regardless of the type of nutritional supplementation, patients receiving dietary supplements experienced a significantly slower loss of muscle tissue and tended to live longer. Many studies have examined the use of nutritional supplementation to counter the effects of cancer cachexia, for example, by adding 'calories ${ }^{\text {,33) }}$ or by using $n-3$ fatty acids ${ }^{(34,35)}$; however, results have been inconclusive and may be secondary to the use of crude body composition tools such as weight or bioelectrical impedance analysis. Using CT imaging, we demonstrated that dietary supplement use is associated with attenuated muscle wasting, and this was related to improved survival in pancreatic cancer patients. Similarly, Mantovani et $a l^{(36)}$ used CT and dual-energy X-ray absorptiometry (DXA) to identify attenuated losses in lean body mass with nutritional supplement. While we found a positive association between dietary supplements and survival in pancreatic cancer patients, the heterogeneity in the type of supplement used, combined with the small number of patients receiving 
supplement ( $n$ 27), prevented further analysis. Thus, future investigations using CT imaging or other precise modalities of body composition to specifically quantify changes in muscle and fat mass are warranted for identifying successful supplements.

Surprisingly, sarcopenic patients lost significantly less muscle tissue at an attenuated rate when compared with non-sarcopenic patients. Sarcopenic patients also tended to lose less VAT and have improved survival outcomes when compared with non-sarcopenic patients. In the present study, sarcopenic patients were diagnosed with earlier-stage cancer when compared with non-sarcopenic patients. The majority of sarcopenic patients ( $42 \%$ ) were diagnosed with stage IIB cancer compared with only $19 \%$ of non-sarcopenic patients $(P=0.008)$. Involuntary weight loss is often one of the first reported symptoms of pancreatic cancer ${ }^{(37)}$; thus, early weight loss may have contributed to their sarcopenia, and may have led to an earlier diagnosis. Patients diagnosed with earlier-stage pancreatic cancer have improved survival outcomes ${ }^{(38)}$, which supports the findings of the present study.

Similar to the findings of Tan et al. ${ }^{(8)}$, sarcopenia alone did not predict poorer survival outcome in pancreatic cancer patients. Tan et $a l .{ }^{(8)}$ observed increased hazard ratios in sarcopenic obese patients when compared with other patients (normal-weight non-sarcopenic, obese non-sarcopenic and normal-weight sarcopenic collectively); however, there were no significant differences between sarcopenic patients and non-sarcopenic patients.

Interestingly, we did not observe any significant influence on different treatment types on body composition. Current literature has demonstrated that patients who undergo surgery experience significant tissue loss ${ }^{(7,39)}$. However, due to the short time course of the disease, the small sample size and heterogeneity in the type of surgery performed, as well as the variability in the timing of the surgery, surgery itself was not significantly related to muscle and adipose tissue loss. Other treatment modalities such as chemotherapy have been related to lean tissue loss ${ }^{(40)}$; however, this could not be evaluated in the present study due to the high proportion (88\%) of patients receiving these treatments.

\section{Limitations of the present study}

CT image analysis and patient history reviews provided a feasible method to evaluate body composition and relationships to clinical parameters in pancreatic cancer patients. We identified associations between clinical outcomes and markers, not causality; however, these associations offer essential considerations to build future clinical studies in nutrition and rehabilitative intervention. The present study also demonstrates the importance of using precise methods to measure body composition. CT image analysis presents a highly precise and reproducible method to evaluate changes in body composition. Although it was not possible to control for the timing of CT images or the timing of clinical measurements, body composition analysis was normalised in a representative manner of the disease trajectory. Also, the small sample size of the present study influenced our ability to identify specific nutritional supplements that may attenuate muscle loss and to further examine the influence of nutrition on body composition. The small sample size and the unknown timing of each surgery during the disease time trajectory limited the interpretation of the surgery's potential influence on muscle and adipose tissue. However, we were still able to demonstrate relationships between body composition change and a number of clinical parameters. The description of changes in body composition in pancreatic cancer and the interrelationships between body composition, clinical markers and survival is fundamental to developing future successful interventions that aim to counter muscle and fat loss in cancer cachexia.

\section{Conclusions}

In conclusion, patients with pancreatic cancer lost significant amounts of muscle and adipose tissue during the course of the disease trajectory; however, the rate at which patients lost VAT and skeletal muscle was a more important indicator of survival than the total amount of tissue loss. The presence of clinical biomarkers associated with cachexia at cancer diagnosis, such as anaemia and diabetes, may exacerbate muscle and adipose tissue loss. Loss of skeletal muscle may be attenuated by dietary supplementation and may prolong survival; however, the type, quantity and timing of dietary supplementation required future investigation.

\section{Acknowledgements}

The authors would also like to acknowledge Roseanna Pressutti, Stephanie Fernandez and Lianne Lin for their technical support. There are no conflicts of interest to disclose. This study received no specific grant from any funding agency in the public, commercial or not-for-profit sectors. K. M. D. S., K. Z., R. K. W., T. C., D. K., G. R. M. and M. M. have contributed to the design of the study; K. M. D. S., L. Y., K. Z., R. K. W., T. C., M. M. have contributed to the data collection and analysis; all authors have contributed to the writing and reviewing of the manuscript. All authors read and approved the final manuscript.

\section{References}

1. Vigano M, Bruera E, Jhangri GS, et al. (2000) Clinical survival predictors in patients with advanced cancer. Arch Intern Med 160, 861-868.

2. Bachmann J, Heiligensetzer $\mathrm{M}$, Krakowski-Roosen $\mathrm{H}$, et al (2008) Cachexia worsens prognosis in patients with resectable pancreatic cancer. J Gastrointest Surg 12, 1193-1201.

3. Fearon K, Strasser F, Anker SD, et al. (2011) Definition and classification of cancer cachexia: and international consensus. Lancet Oncol 12, 489-495.

4. Wigmore SJ, Plester CE, Richardson RA, et al. (1997) Changes in nutritional status associated with unresectable pancreatic cancer. Br J Cancer 75, 106-109.

5. Wang X, Hu Z, Hu J, et al. (2006) Insulin resistance accelerates muscle protein degradation: activation of the ubiquitinproteasome pathway by defects in muscle cell signaling. Endocrinology 147, 4160-4168.

of the present study influenced our ability to identify specific 
6. Evans WJ, Morely JE, Argiles J, et al. (2008) Cachexia: a new definition. Clin Nutr 27, 793-799.

7. Liedman B, Andersson H, Bosaeus I, et al. (1997) Changes in body composition after gastrectomy: results of a controlled, prospective clinical trial. World J Surg 21, 416-421.

8. Tan BHL, Birdsell LA, Martin L, et al. (2009) Sarcopenia in overweight or obese patients is an adverse prognostic factor in pancreatic cancer. Clin Cancer Res $\mathbf{1 5}$, 6973-6973.

9. Despres JP \& Lemieux I (2006) Abdominal obesity and metabolic syndrome. Nature 444, 881-887.

10. Johansen D, Stocks T, Jonsson H, et al. (2010) Metabolic factors and the risk of pancreatic cancer: a prospective analysis of almost 580,000 men and women in the metabolic syndrome and cancer project. Cancer Epidemiol Biomarkers Prev 19, 2307-2317.

11. Balentine CJ, Enriquez J, Fisher W, et al. (2010) Intra-abdominal fat predicts survival in pancreatic cancer. J Gastrointest Surg 14, 1832-1837.

12. World Health Organization (2001) Iron Deficiency Anaemia: Assessment, Prevention, and Control. Geneva: World Health Organization. http://whqlibdoc.who.int/hq/2001/WHO_NHD_ 01.3.pdf (WHO/NHD/0.1.3).

13. Shen W, Punyanitya M, Wang Z, et al. (2004) Total body skeletal muscle and adipose tissue volumes; estimates form a single abdominal cross-sectional image. J Appl Physiol 97, 2333-2338.

14. Shen W, Punyanitya M, Wang Z, et al. (2004) Visceral adipose tissue relations between single-slice areas and total volume. Am J Clin Nutr 80, 271-278.

15. Mourtzakis M, Prado CMM, Lieffers JR, et al. (2008) A practical and precise approach to quantifications of body composition in cancer patients using computed tomography images acquired during routine care. Appl Physiol Nutr Metab 33, 997-1006.

16. Alberti G, Zimmet P, Shaw J, et al. (2006) The IDF consensus worldwide definition of the metabolic syndrome. International Diabetes Federation. http://www.idf.org/ webdata/docs/IDF_Meta_def_final.pdf.

17. Mitsiopoulos N, Baumgartner RN, Heymsfield SB, et al. (1998) Cadaver validation of skeletal muscle measurement by magnetic resonance imaging and computerized tomography. J Appl Physiol 85, 115-122.

18. Miller KD, Jones E, Yanovski JA, et al. (1998) Visceral abdominal-fat accumulation associated with use of indinavir. Lancet 351, 871-875.

19. Snyder WS, Cooke MJ, Manssett ES, et al. (1975) Report of the Task Group on Reference Man. Oxford: Pergamon.

20. World Health Organization (2001) Obesity: Preventing and Managing the Global Epidemic. WHO Technical Report Series no. 894. Geneva: WHO.

21. Frontera WR, Hughes VA, Fielding RA, et al. (2000) Aging of skeletal muscle; a 12-year longitudinal study. J Appl Physiol 88, 1321-1326.

22. Lexell J, Taylor CC \& Sjostrom M (1988) What is the cause of the aging atrophy? Total number, size, and proportion of different fibre types in whole vastus lateralis from 15- to 83-year-old men. J Neurol Sci 84, 275-294.
23. Lieffers JR, Mourtzakis M, Hall KD, et al. (2009) A viscerally driven cachexia syndrome in patients with advanced colorectal cancer; contributions of organ and tumor mass to whole-body energy demands. Am J Clin Nutr 89, 1173-1179.

24. Hallgreen CE \& Hall KD (2008) Allometric relationship between changes of visceral fat and total mass. Int $J$ Obes (Lond) 32, 845-852

25. Bruckart K, Beca S, Urban RJ, et al. (2010) Pathogenesis of muscle wasting in cancer cachexia: targeted anabolic and anti-catabolic therapies. Curr Opin Clin Nutr Metab Care 13, 410-416.

26. Glass DJ (2010) Signaling pathways perturbing muscle mass. Curr Opin Clin Nutr Metab Care 13, 225-229.

27. Tayek JA (1992) A review of cancer cachexia and abnormal glucose metabolism. J Am Coll Nutr 11, 445-456.

28. Park SW, Goodpaster BH, Lee JS, et al. (2009) Health, aging, and body composition study. Excessive loss of skeletal muscle mass in older adults with type 2 diabetes. Diabetes Care 32, 1993-1997.

29. Kim TN, Park MS, Yang SJ, et al. (2010) Prevalence and determinant factors of sarcopenia in patients with type 2 diabetes: the Korean Sarcopenic Obesity Study (KSOS). Diabetes Care 33, 1497-1499.

30. Kahl J, Wykes SM, Russell ST, et al. (2005) Expression of the ubiquitin-proteasome pathway and muscle loss in experimental cancer cachexia. Br J Cancer 93, 774-780.

31. Weiss G \& Goodnough LT (2005) Anemia of chronic disease. $N$ Engl J Med 352, 1011-1023.

32. Rasic-Milutinovic Z, Perunicic-Pekovic G, Ristic-Medic D, et al. (2009) Insulin resistance and chronic inflammation are associated with muscle wasting in end-stage renal disease patients on hemodialysis. Gen Physiol Biophys 28, 184-189 (Spec No).

33. Morley JE (2009) Calories and cachexia. Curr Opin Clin Nutr Metab Care 12, 607-610.

34. Colomer R, Moreno-Nogueira JM, Garcia-Luna PP, et al. (2007) n-3 Fatty acids, cancer and cachexia: a systematic review of the literature. Br J Nutr 97, 823-831.

35. Brown TT, Zelnik DL \& Dobs AS (2003) Fish oil supplementation in the treatment of cachexia in pancreatic cancer patients. Int J Gastrointest Cancer 34, 143-150.

36. Mantovani G, Maccio A, Madeddu C, et al. (2010) Randomized phase III clinical trial of five different arms of treatment in 332 patients with cancer cachexia. Oncologist 15, 200-211.

37. Holly EA, Chaliha I, Bracci PM, et al. (2004) Signs and symptoms of pancreatic cancer: a population-based case-control study in the San Francisco Bay area. Clin Gastroenterol Hepatol 2, 510-517.

38. Wasif N, Ko CY, Farrell J, et al. (2010) Impact of tumor grade on prognosis in pancreatic cancer: should we include grade in AJCC staging? Ann Surg Oncol 17, 2312-2320.

39. Kiyama T, Mizutani T, Okuda T, et al. (2005) Postoperative changes in body composition after gastrectomy. J Gastrointest Surg 9, 313-319.

40. Awad S, Tan BH, Cui H, et al. (2011) Marked changes in body composition following neoadjuvant chemotherapy for oesophagogastric cancer. Clin Nutr 31, 74-77. 